\title{
PERCEPÇÃO DAS MULHERES SOBRE A EXPERIÊNCIA DO PRIMEIRO PARTO: IMPLICAÇÕES PARA O CUIDADO DE ENFERMAGEM
}

\author{
WOMEN'S PERCEPTION OF THEIR FIRST LABOR EXPERIENCE: \\ IMPLICATIONS FOR NURSING
}

\section{PERCEPCIÓN DE LAS MUJERES EN LA EXPERIENCIA DEL PRIMER PARTO: IMPLICACIONES PARA ENFERMERÍA}

\author{
Carolyn CRistina Reis \\ Karla Romana Ferreira de Souza ${ }^{* *}$ \\ Danielle Santos Alves ${ }^{* * *}$ \\ Inez Maria TenÓRIO ${ }^{* * *}$ \\ WALDEMAR BRANDÃo Neto ${ }^{* * * *}$
}

\begin{abstract}
RESUMO
Objetivou-se estudar a percepção das mulheres sobre o primeiro parto no contexto obstétrico de uma maternidade do Recife. Método: Trata-se de uma pesquisa descritiva e qualitativa. Foram entrevistadas 10 primíparas internadas no Alojamento Conjunto de um Hospital Universitário de Pernambuco, durante três meses. A coleta de dados foi realizada por um roteiro de entrevista semiestruturado. Os dados foram submetidos à análise de conteúdo, modalidade temática, e interpretados segundo a Teoria do Cuidado Transpessoal de Jean Watson. Resultados: As mulheres relataram a criação do vínculo com a equipe de saúde e a satisfação do primeiro contato com o filho. Possuíam déficit de conhecimento sobre sua situação de saúde e não se sentiam confortáveis para participar das decisões no cuidado. Conclusão: Há persistência do intervencionismo conservador nas memórias das primíparas, revelando ainda a necessidade de discussões acerca do tema, que permita a construção de modelos de cuidado em Enfermagem favoráveis a humanização e a autonomia.
\end{abstract}

Palavras chave: Saúde da mulher, humanização da assistência, parto humanizado, enfermagem obstétrica, cuidados de enfermagem.

\section{ABSTRACT}

Objective: To study the perception of primiparous women in the obstetric context of a maternity hospital in

\footnotetext{
* Enfermeira. Especialista em Enfermagem na Saúde da Mulher pelo Hospital das Clínicas da Universidade Federal de Pernambuco, Enfermeira Assistencial do Hospital Universitário da Universidade Federal de Alagoas. Maceió-AL, Brasil. Email: carolyncristina@hotmail.com

** Enfermeira. Doutoranda do Programa Associado de Pós-Graduação em Enfermagem da Universidade de Pernambuco e Universidade Estadual da Paraíba, Coordenadora do curso de graduação em Enfermagem da Faculdade Integrada de Pernambuco. Recife-PE, Brasil. Email: karlaromana@ig.com.br

*** Enfermeira. Doutoranda do Programa de Pós-Graduação em Ciências da Computação da Universidade Federal de Pernambuco, Professora Assistente do Departamento de Enfermagem da Universidade Federal de Pernambuco. Recife-PE, Brasil. Email: angeldannyalves@gmail.com

${ }^{* * * *}$ Enfermeira. Doutora em Serviço Social pela Universidade Federal de Pernambuco, Professora Adjunta do Departamento de Enfermagem da Universidade Federal de Pernambuco. Recife-PE, Brasil. Email: inezmariatenorio@gmail.com

${ }_{* * * * *}$ Enfermeiro. Doutorando no Programa de Pós-Graduação em Saúde da Criança e do Adolescente pela Universidade Federal de Pernambuco, Professor Assistente da Faculdade de Enfermagem Nossa Senhora das Graças (FENSG) da Universidade de Pernambuco. Recife-PE, Brasil. Email: waldemar.neto@upe.br
} 
Recife. Method: This is a descriptive and qualitative research. Ten primiparous women admitted at a University Hospital of Pernambuco were interviewed for three months. The instrument of data collection was a semistructured interview. After full transcription, the speeches were subjected to content analysis, thematic modality, and later interpreted according to Jean Watson's Theory of Transpersonal Care. Results: Women reported having created a bond with the health team and feeling satisfaction from the first contact with their child. They had lack of knowledge about their health condition and could not participate in decisions made about their health. Conclusion: There is persistence of conservative interventionism in the memories of their first delivery, thus revealing the need for further discussion about the subject and the construction of nursing care models in favor of humanization and autonomy.

Key words: Women's health, humanization of assistance, humanized delivery, obstetric nursing, nursing care.

\section{RESUMEN}

Objetivo: Estudiar la percepción de las mujeres en el primer parto en el contexto obstétrico de una maternidad de Recife. Material y método: Se trata de un estudio descriptivo y cualitativo. Fueran entrevistadas 10 primíparas ingresadas en el alojamiento conjunto de un Hospital Universitario de Pernambuco, durante tres meses. La recolección de datos se realizó mediante una guía de entrevista semiestructurada. Los datos se sometieron a análisis de contenido, modalidad temática, y se interpretan de acuerdo con la Teoría del Cuidado Transpersonal de Jean Watson. Resultados: Las mujeres informaron de la creación del vínculo con el equipo de salud y la satisfacción del primer contacto con el niño. Ellas tenían poco conocimiento sobre su situación de salud y no pudieron participar en las decisiones. Conclusión: Hay persistencia de intervencionismo conservador en los recuerdos del primer parto, lo que revela la necesidad de discusión sobre el tema, que permite la construcción de modelos de atención de Enfermería en favor de la humanización y la autonomía.

Palabras clave: Salud de la mujer, humanización de la asistencia, parto humanizado, enfermería obstétrica, cuidados de enfermería.

Fecha recepción: 30/03/15 Fecha aceptación: 28/10/16

\section{INTRODUÇÃO}

A garantia de vivenciar plenamente a gestação, o parto e pós-parto é dever dos profissionais de saúde comprometidos com a qualidade do nascimento (1). Para disseminar a proposta da humanização, tem-se buscado ampliar as ações de qualificação profissional, pois a assistência à saúde desqualificada e a ausência de acompanhamento profissional são fatores que causam percepções negativas sobre o parto (2). Os profissionais que atuam distanciados das mulheres assistidas, não conseguem perceber suas reais necessidades (3).

Geralmente, puérperas se referem ao parto enfatizando medo, dor e insegurança, compondo uma condição de vulnerabilidade e dependência. A modernização da obstetrícia substituiu o protagonismo da mulher e culminou na modificação do cenário e da cena. O hospital passou a ser o ambiente mais seguro e o profissional de saúde, o ator principal. Com o desenvolvimento obstétrico, o contexto da parturição foi vinculado à mulher incapaz de parir sem utilizar-se das tecnologias. Trata-se a parturição como um problema de saúde ou um evento meramente biológico, desconsiderando que o parto constitui uma experiência que ocorre em um contexto biossociocultural (4).

Em contrapartida, a Teoria do Cuidado Transpessoal de Jean Watson corrobora com 
a ideia de que o cuidado transpassa a perspectiva da relação meramente técnica e cumpridora de tarefas entre o enfermeiro e a mulher assistida. Nesse contexto, o profissional de Enfermagem relaciona-se a partir da ótica humanista, considerando não somente a matéria, como também estabelecendo um relacionamento intersubjetivo, indo de encontro com a visão tecnocêntrica da parturição (5).

Reconhece-se que os avanços tecnológicos muito contribuíram para a redução da morbimortalidade materna e neonatal. Porém, a assistência passou a ser mecanizada, assimétrica e desumanizada, gerando parturientes manipuladas e desinformadas (3). Esta realidade permite gerar algumas indagações: o modelo de atenção ao parto vem acompanhando as tendências de modificações das relações na produção do cuidado em saúde e enfermagem? A presença de maternidades e centros obstétricos dentro de uma estrutura rígida hospitalar, coexistindo com o modelo biomédico, pode inviabilizar as transformações desejadas pela via da humanização? E por fim, até que ponto os profissionais de saúde estão abertos a dividir seu espaço de poder com as mulheres nas negociações do cuidado?

Diante do exposto, este estudo oportuniza aos profissionais de saúde a adaptação da abordagem às mulheres de acordo com a análise de como a percepção sobre o primeiro parto está sendo construída pelas primíparas durante a vivência parturitiva. Desse modo, objetivou-se estudar a percepção das mulheres sobre o primeiro parto no contexto obstétrico de uma maternidade pública do Estado de Pernambuco, Brasil.

\section{MATERIAL E MÉTODO}

Trata-se de uma pesquisa descritiva com abordagem qualitativa. Foram selecionadas 10 primíparas internadas no Alojamento Conjunto de um Hospital Universitário de
Pernambuco, de abril a junho de 2014, utilizando amostragem do tipo conveniência e definição do número de participantes foi realizada por procedimentos de saturação teórica (6). A seleção das participantes obedeceu aos seguintes critérios: primíparas de parto normal ou cesáreo, realizado no Centro Obstétrico da instituição, e que se encontravam a partir do primeiro dia pós-parto. Foram excluídas as puérperas que apresentaram complicação clínica que inviabilizasse a pesquisa ou possuíam limitações para comunicação verbal.

A coleta de dados aconteceu durante o período da tarde e principiou após a assinatura do Termo de Consentimento Livre e Esclarecido. Para tanto, foi realizada entrevista gravada mediante aplicação de um roteiro semiestruturado, abordando cinco questões norteadoras a cerca da experiência do parto e por questões fechadas, para caracterização do perfil sociodemográfico. As entrevistas foram realizadas por uma das autoras, que não possuía envolvimento com a assistência prestada, e em média duraram 4 minutos.

As questões norteadoras foram: "durante a gravidez, qual tipo de parto você desejava?"; "por que você escolheu este parto?"; "no pré-natal, você recebeu orientação de qual profissional de saúde sobre o parto? $\mathrm{O}$ que ele lhe disse a respeito deste parto? E do outro tipo de parto?"; "como foi para você a experiência do parto?"; "qual sua opinião sobre os profissionais envolvidos no seu parto? E sobre o local onde ele ocorreu?".

Após a transcrição integral das entrevistas, o conjunto de dados foi submetido a tratamento e análise, por meio da técnica de análise de conteúdo, modalidade temática, executando-se as seguintes etapas: pré-análise, exploração do material, tratamento dos dados e inferência dos resultados. Na pré-análise foi realizada a leitura flutuante das entrevistas, destacando-se os pontos chave, seguido da exploração do material com a leitura compreensiva e exaustiva de todo o conteúdo. Após, realizou-se a codificação das mensagens, por 
meio da qual se apreenderam os núcleos de sentido, os quais foram agrupados, gerando-se as categorias temáticas. Finalizada a categorização, realizou-se a inferência a partir dos dados obtidos, considerando o contexto da linguagem, a condição do emissor e suas significações (7).

A síntese interpretativa dos dados ocorreu à luz da Teoria do Cuidado Transpessoal de Jean Watson e dos documentos ministeriais referente às políticas de humanização e da assistência integral à saúde da mulher.

As participantes foram identificadas com nomes fictícios, para assegurar o anonimato e a confidencialidade. O estudo foi aprovado pelo Comitê de Ética em Pesquisa do Centro de Ciências da Saúde da Universidade Federal de Pernambuco, com protocolo no 554.376 e registro CAAE 26181913.2.0000.5208.

\section{RESULTADOS}

\section{Caracterização das participantes}

As 10 primíparas que participaram estavam na faixa etária de 19 a 38 anos. As puérperas menores de 18 anos não participaram, pois não estavam durante a coleta acompanhadas por seus representantes legais. Cinco delas possuíam ensino fundamental, 4 ensino médio e 1 ensino superior. Cinco mulheres relataram ser solteiras e cinco responderam estar casadas, sendo que apenas uma casada e duas solteiras eram independentes financeiramente.

\section{Experiência antes do parto, do parto e do pós-parto}

A maioria das participantes verbalizou que durante a gestação preferia o parto normal, enquanto que duas delas apresentaram preferência pelo parto cesáreo e uma não expressou preferência a nenhum tipo de parto. Aquelas mulheres que expressaram suas preferências pelo parto normal, o fizeram pela percepção de que a recuperação do parto normal é menos prolongada e trabalhosa que a do pós-parto cesáreo. Além de que no pós-parto normal, a mulher precisa de menos ajuda para realizar suas atividades. Angelita, que preferia o parto normal e conseguiu passar por essa experiência, disse:

Porque é muito trabalho [pós-parto cesáreo], minha filha... Oxe! Pra você se levantar, tomar um banho, tudo você precisa de ajuda. E normal... precisa só naquele momento, depois... (Angelita).

Outra mulher, que preferia o parto normal e foi submetida ao parto cesáreo, explicou sua escolha:

É mais demoroso a recuperação no parto cesariano... Mais demoroso, mais complicado (Selenita).

As mulheres verbalizaram como imaginavam o parto enquanto estavam gestantes. A dor do parto fez parte do imaginário delas, como se percebe no comentário abaixo, que associa a dor de parir com a morte, baseada em uma influência sociocultural. Sua fala refletiu a preferência pelo parto cesáreo, porém a mesma vivenciou o parto normal:

Porque eu tinha medo de normal. Porque o povo falava muito. Dizia que era a dor da morte. Muita gente dizia que... eu fiquei sabendo que no sertão uma mulher teve três normal, aí ela não sobreviveu não. Foi, ela morreu de parto e os bebê ficou (Ágata).

Nota-se na fala de Ágata que a construção da percepção do medo da dor e de vivenciar o processo do parto normal está relacionado ao sofrimento, à morte da mulher e à possibilidade de deixar o filho sem mãe. Outra participante, que também preferia o parto cesáreo e não passou por essa experiência, vinculou seu medo do parto normal a uma 
possibilidade de passar mal e não ter coragem, o que reflete a insegurança de ser capaz de parir. Observa-se também a ausência de confiança no próprio corpo:

Ah... eu tinha muito medo de ter normal. Medo de acontecer alguma coisa, assim... de passar mal, né... É... não sei, da falta de coragem também... Do medo, dói... (Jaspe).

Em outro momento, a depoente Ágata admite o medo de a criança não evoluir tranquilamente durante o trabalho de parto, influenciada pelo comentário do profissional médico:

Aí o médico da ultrassom disse que... é... se eu... fosse por ele, eu não teria normal, era cesárea porque às vezes o bebê fica enganchado. Aí era por isso também que eu tinha medo (Ágata).

Cristal refere a segurança que sentiu ao ser informada sobre a realização do parto cesáreo, já que sabia que o feto estava em sofrimento. Observa-se o conhecimento da sua situação clínica como condição significativa para construção da percepção:

Eu estava perdendo muito líquido, meu bebê estava sem oxigênio. Então quando disse que ia ser cesáreo para mim foi um alívio, né... [...] E porque seria mais rápido também, né... Porque ele já estava sofrendo na barriga... (Cristal).

As mulheres se depararam com reforços condizentes e contrários a sua preferência, realizados pelos profissionais, durante o prénatal. Houve relatos em que a enfermeira realizou uma orientação que tendeu para a preferência ao parto normal. Ágata, que iniciou seu pré-natal no primeiro trimestre e passou pelo parto normal, contrariando sua preferência, completou dizendo que a médica havia indicado uma tendência para o parto cesáreo:

A enfermeira queria que eu... fosse por ela, eu tivesse normal... que a recuperação era melhor. E a doutora, cesárea (Ágata).

Pérola, que vivenciou o parto de sua preferência (normal) e deu início ao pré-natal no segundo trimestre, disse:

[A enfermeira] Disse que era bom... normal... a recuperação era melhor... tanto pra mim quanto pro bebê, né. Falou que era bom também [parto cesáreo], mas... se fosse normal seria melhor ainda (Pérola).

Chamou atenção um quadro de déficit de conhecimento entre as mulheres sobre sua condição clínica, que constituiu uma situação geradora de angústia. Isso pode ser notado no caso de uma das mulheres, em que além do desconhecimento sobre a evolução clínica e das perspectivas para o parto, observa-se uma tentativa sem êxito de esclarecer suas dúvidas:

Eles não diziam que ia ser normal ou que ia ser cesáreo... Eu perguntava e 'eu não sei', 'eu não sei... Aí eu ficava com mais medo ainda... Meu Deus... Aí, 'não tem como seu filho nascer normal?', aí 'não'... 'vai tirar cesáreo?' '.. 'não sei'. Se não tinha como normal, porque não ia tirar cesáreo? Aí eu ficava com medo, será que meu filho não vai nascer? (Safira).

Uma entrevistada, que preferia parto normal e foi submetida ao parto cesáreo, comentou de modo crítico o tempo de espera para a resolução de seu caso e revelou que sua opinião não foi considerada ao expressar que não tinha condição de enfrentar o parto normal. Nota-se que ela não assumiu o papel de protagonista do processo, visto que não participou das decisões sobre seu caso:

Eu não gostei tanto não [dos profissionais], porque sei lá... Viram que eu tava assim sofrendo muito e esperou muito pra ter cesáreo... Não tinha condição de eu ter normal. Ai ficaram esperando (Cristal). 
A assistência prestada precisa corresponder às necessidades das parturientes. As entrevistadas se mostraram incomodadas com os procedimentos, sem serem previamente informadas sobre como colaborar. Relataram posturas profissionais controladoras diante de suas necessidades emocionais, afetivas e culturais:

Só o médico lá que eu achei meio ruim, né... Teve uma hora que ele forçou um pouco a, pra nascer a segunda e... mas foi tranquilo. Doeu um pouco aí eu...'para um pouquinho, pera aí, pere aí, pere aí... Aí ele forçou mais um pouco e tirou e depois ela saiu... (Jaspe).

Uma das mulheres, refletindo sobre seu processo de parturição, relembrou o desconforto dos toques vaginais, o que transparece uma prática em que os profissionais decidem o que vão realizar no corpo da parturiente:

Pelo o que passei, né... Assim... A dor que eu senti dos toque, né... (Safira).

A satisfação com a assistência ao parto está relacionada com a interação com a equipe, como expressado abaixo. As orientações e o apoio emocional foram percebidos por elas e contribuíram para que se sentissem mais tranquilas. Verifica-se a necessidade da construção do vínculo entre parturiente e profissional, para que haja satisfação com a assistência:

Pra mim... tá de parabéns! Porque eles me tranquilizaram... Eu cheguei lá nervosa... Aí... mas eles foi conversando... ai eu fiquei mais calma (Jade).

Eu gostei do pessoal que fez minha cirurgia, eu gostei muito. Assim, conversava, orientava, mandando ter calma, o que podia acontecer... (Cristal).

As percepções das mulheres sobre o primeiro parto também alcançaram a ocasião em que tiveram o primeiro contato com os recém-nascidos. Uma das mulheres mencionou o contato com o filho, como o melhor momento vivenciado:

Eu gostei muito de escutar minha filha chorar, né... pela primeira vez... fiquei emocionada... Foi, ela não saiu de perto de mim não... Quando tiraram, colocaram ela perto de mim até ela ir pra sala... Eu gostei mais dessa parte (Safira).

Por vezes, as situações impedem tal vivência, como no caso abaixo em que uma das mulheres esclareceu que não teve contato prolongado com suas filhas após o nascimento:

Foram pra sala dos neném, né... Tiraram, eu fiquei na outra sala, né... e levaram ela (Jaspe).

\section{Experiência durante a passagem pelo centro obstétrico}

Nesta categoria temática as mulheres expressaram suas percepções a respeito do Centro Obstétrico. Emergiram das suas falas conflitos atrelados à quantidade excessiva de mulheres internadas. A inserção da mulher na unidade gerou uma discordância de expectativas quanto à capacidade de leitos esperada e a alta demanda, que refletiram na organização do serviço e estão relacionados à atuação profissional.

Uma das entrevistadas lamenta ter sido internada em uma maca. Percebeu que havia no serviço mais pacientes que a estrutura e os recursos humanos poderiam receber e refletiu que a demanda excessiva prejudicou a qualidade da assistência a elas próprias:

No início, foi um pouco ruinzinho porque eu ficava por cima de maca, ficava esperando, muita gente, essas coisas, entendeu... (Cristal).

E ainda complementa:

Olha assim... eu não sei se é porque tem muita 
gente também, né... Porque, assim no dia que cheguei aqui era muitas grávida... Então isso também prejudica as pessoas de ta trabalhando... É muita gente pra dar conta... (Cristal).

Quanto ao ambiente hospitalar, os relatos evidenciaram satisfação com a estrutura do centro obstétrico. A limpeza, a organização e a presença dos materiais necessários foram percebidas pelas puérperas:

Muito bom. Tudo muito organizado, limpinho... (Turmalina).

Tudo esterilizado, tudo bem organizado, esperando só para minha chegada. Tudo bem organizado, material não faltou... (Esmeralda).

As mulheres também comentaram sobre o fato de compartilharem o ambiente com outras pessoas. Uma delas enfatizou que se sentiu incomodada:

Eu não gostei muito não porque via as outras meninas sentindo dor, contração... Ficava com medo e minha pressão subia (Safira).

Já outra entrevistada encontrou vantagem em dividir a expectação:

Muito bom porque só assim você pega experiência de cada uma (Turmalina).

\section{DISCUSSÃO E CONCLUSÃO}

Frente aos resultados apresentados na categoria "experiência antes do parto, do parto e do pós-parto”, no que diz respeito à preferência da mulher ao parto, notam-se dificuldades para a concretização do desejo pelo tipo de parto, mesmo naquelas mulheres que detinham o conhecimento popular sobre esta experiência. Assim, pode-se afirmar que o corpo feminino incapaz de passar pelo processo de parturição sem o controle tecnoló- gico é consequência da dependência biomédica do parto (4).

Faz-se necessário observar que, por ser uma instituição referência de serviço terciário, recebendo encaminhamentos para partos de alto risco, esperava-se uma quantidade significativamente maior de mulheres que vivenciaram o parto cesáreo, contudo, das dez primíparas, quatro estavam em pós-parto normal.

Em um estudo comparativo sobre a escolha do parto com 100 gestantes, verificou-se que $60,7 \%$ das nulíparas e 70,5\% das primíparas preferiam parto normal. Concluiu-se que não existe diferença significativa acerca da vivência e da escolha do parto entre as mulheres que já possuíam experiência e as que não possuíam (8).

Um estudo nacional com 23.940 puérperas obteve $66 \%$ de participantes com preferência para o parto normal, 26,7\% para o parto cesáreo e as demais sem preferência no início da gestação. A maioria das mulheres que preferiram o parto cesáreo recebeu assistência privada (9).

A preferência pelo parto normal se relaciona à capacidade plena de realização do autocuidado no pós-parto e à possibilidade de recuperação rápida e indolor. A dor pósoperatória do parto cesáreo está associada à recuperação prolongada, causando dependência e postergando o retorno às atividades de vida diárias (3).

Nos relatos apresentados por Ágata e Jaspe sobre a percepção da dor, verifica-se que esta vivência foi construída no decorrer das gerações. Diversas vezes, o resultado gerado por essa transmissão de experiências é o medo, principalmente naquelas que nunca passaram pelo parto normal. A dor relaciona-se com o sofrimento, prejudicando a forma de lidar com o trabalho de parto (10). Neste cenário, o papel do profissional de Enfermagem ganha importância, pela capacidade de ofertar informações seguras às mulheres, estimulando sua consciência crítica diante das possibilidades de escolha. 
O conceito de reciprocidade do cuidado, destacado por Watson, aproxima enfermeira e mulher para o atendimento de suas necessidades biopsicossociocultural e espiritual. De modo a firmar um compromisso com a qualidade da assistência à mulher durante o parto e alcance de um cuidado ético, moral e humano, valorizando sobremaneira a relação interpessoal cuidador/ser cuidado (5).

Ainda no depoimento de Ágata, foi revelado um desencorajamento da mulher, pelo médico, ao colocar o parto cesáreo como um mecanismo seguro de salvar vidas e de um nascimento saudável. A institucionalização do parto revela o receio de possíveis complicações clínicas. Nesse processo, esteve envolvido o medo do prejuízo à integridade da vida do filho, da morte dele e da própria morte (3).

A interrupção da gestação foi compreendida pelas mulheres considerando como prioridade a segurança para o nascimento do filho. Mesmo quando o parto vivenciado não é o escolhido previamente, como ocorreu com Cristal, que preferia o parto normal, obtêm-se a satisfação. A preservação da vida da criança supera a preferência da mãe sobre a via de parto (8).

Notou-se que parte das preferências por determinado tipo de parto possuiu influência profissional vinda do pré-natal. Em um estudo com 11 médicos obstetras, verificouse que apenas $27,3 \%$ concordaram que a gestante do sistema público de saúde deveria ter o direito de escolher a via de parto, ideia contrária à refletida no relato de Ágata sobre a ocasião com o médico que realizou sua ultrassonografia (11).

A partir dos depoimentos das mulheres, identificaram-se discursos contrários da enfermeira e do médico no momento do prénatal, enfatizando uma descontinuidade da assistência e fragilidade de uma escuta ativa na consulta. Esta atitude concorre para a prática de uma conduta impositiva, onde o profissional "dono do saber científico" decide o que fazer e a melhor escolha a tomar.
As gestantes carregam consigo as próprias experiências, inseguranças e incertezas e o profissional de saúde possui sua visão sobre os eventos envolvidos com a gestação e o parto. Esse encontro é marcado pela hegemonia do profissional, constituindo forte influência na escolha (12). As relações de poder, existentes nas práticas em saúde, servem de obstáculo para que as mulheres sejam protagonistas do parto, além disso, reafirmam a opressão sobre o corpo e naturalizam práticas inadequadas.

Cabe ressaltar, que a teoria de Watson possui foco no ser humano, ou seja, o desenvolvimento de ações de cuidado que integram as dimensões do ser humano: corpo, mente e espírito. O que possibilita ao enfermeiro reconhecer a complexidade humana e amplia sua visão profissional, trazendo para o contexto da assistência obstétrica opções de cuidado direcionadas ao atendimento integral (5).

Estudo realizado com 11 médicos obstetras constatou que $63,6 \%$ dos pesquisados possuíam preferência pelo parto cesáreo, porém 100\% dos entrevistados responderam que, se questionados pela gestante sobre qual a melhor via, indicariam o parto natural. $54,5 \%$ afirmaram abordar a escolha do parto no primeiro trimestre e o mesmo quantitativo admitiu que, se a mulher relatasse escolha pelo parto cesáreo, concordariam imediatamente (11).

A assistência prestada deve estar baseada nas necessidades individuais. Informar sobre a situação de saúde contribui para que a mulher participe das decisões. É de grande importância garantir autonomia, para que ela possa escolher, aceitar ou recusar os procedimentos propostos, mesmo sabendo que a recusa nem sempre é possível nas situações circunstanciais (4).

A violação do direito de escolha e informação sobre a assistência prestada associada ao não envolvimento da mulher como colaborada fez parte da vivência crítica dessas participantes. A necessidade de intervenções 
exige explicação sobre o que será realizado no corpo delas. Pois caso esta realidade não seja percebida e discutida, incorre-se no risco de uma prática de violência obstétrica.

Os procedimentos realizados durante o parto normal intensificam a dor, como relatado pelas mulheres. A medicalização do parto reflete a visão de que o corpo feminino é um instrumento de trabalho, desconsiderando a individualidade e contribuindo para um processo traumático (3). Nota-se que o controle do trabalho de parto e parto por protocolos institucionais prejudicam a participação ativa da mulher e gera insatisfação. A humanização da assistência preconiza considerar as decisões da parturiente, sua autonomia e privacidade (4).

O estabelecimento da comunicação, do diálogo, do acolhimento como tecnologias relacionais sinalizam para os profissionais da saúde o resgate destas atividades também no cenário hospitalar. A educação em saúde fortalece os espaços emancipatórios do cuidado. $\mathrm{O}$ reconhecimento dos saberes do outro pelos profissionais, fornece as bases humanísticas para pensar o cuidado em sua plenitude. $O$ profissional não deve se sentir confrontado pelo paciente, quando este o questiona ou solicita informações de seu interesse, pelo contrário, deve estimular a troca de saberes como ferramenta de crescimento profissional e aprimoramento/manutenção das ações de cuidado (13).

Orientar reflete uma assistência cuidadosa. Quando a mulher sente-se cuidada, adquire segurança para parir. Pois, não se teme apenas a dor, mas também o acolhimento no ambiente hospitalar (10). Na sua vulnerabilidade, a mulher precisa de uma escuta ativa. Há casos em que é maior a necessidade de sensibilidade do profissional que a presença de tecnologias (4).

O contato da mulher com filho, logo após o nascimento, foi revelado pelas mulheres como um momento único, desencadeando sensações diversas dentro do contexto biopsicossociocultural de cada uma delas, com sentimentos e significados favoráveis ao vínculo mãe-filho (14). Após o parto, a mulher passa por uma transição que compreende o alívio da dor e principalmente o reconhecimento do valor do filho, que é percebido como resultado do sucesso dela (10).

O contato íntimo com o filho logo após o nascimento supera os eventos negativos vivenciados ao passo que acontece como um momento compensador (15). Além de que promove um momento de reconhecimento e identificação, em que a mulher percebe-se como mãe (16). Reconhecer a necessidade das mulheres e a experiência que atravessa, pode potencializar transformações no cenário do cuidado da enfermagem obstétrica.

Por outro lado, foi evidenciado no relato de Jasper o incômodo pelo afastamento do bebê nos primeiros momentos após o parto. Esse rompimento no vínculo foi também destacado por outro estudo, o qual menciona que esta situação ocorre, mesmo com a solicitação da mulher para ver seu filho (17). O que gera uma experiência negativa em relação ao parto transpelviano e insatisfação com a assistência.

$\mathrm{Na}$ categoria "experiência durante a passagem pelo centro obstétrico", sobre os depoimentos das mulheres relativos à estrutura e satisfação com o serviço, nota-se que a percepção sobre a demanda excessiva é secundária à percepção da sua influência na atenção profissional. As mulheres refletem sobre essa questão, após se depararem com uma assistência fragilizada e de pouco acolhimento. Este é um problema da perspectiva de quem assiste e de quem está sendo assistido. Portanto, é necessário que os serviços ofereçam as mínimas condições de atendimento a essas mulheres. A atenção oferecida pela equipe contribui para a percepção positiva do atendimento (18).

A Portaria no 1020, de 29 de maio de 2013 do Ministério da Saúde dispõe sobre critérios para implantação e habilitação dos serviços de referência da Atenção à Saúde na Gestação de Alto Risco conforme a Rede Cegonha e 
informa ser necessário que o ambiente compartilhado pelas mulheres esteja adequado às normas estabelecidas pela Agência Nacional de Vigilância Sanitária (19). É necessário que o ambiente seja silencioso e limpo para promover o melhor acolhimento e conforto à parturiente (20).

Um estudo nacional com 15.688 mulheres buscou conhecer a satisfação com o atendimento ao parto, considerando a privacidade. Concluiu que mulheres de cor parda ou preta e em trabalho de parto possuíram menos chances de ter privacidade. Ser atendida pelo setor privado, possuir nível de escolaridade maior, vivenciar o parto cesáreo e ter acompanhante foram fatores relacionados à maior privacidade. A garantia da privacidade auxilia na diminuição do estresse e compõe um dos critérios relacionados à boa avaliação da assistência (18).

Com este estudo evidencia-se que a percepção sobre o primeiro parto está sendo construída, durante a vivência parturitiva, de forma conflituosa pelas primíparas, marcada principalmente pela violação do protagonismo da mulher e pelo desrespeito ao parto enquanto evento fisiológico. Conhecer esta percepção contribui para o entendimento da necessidade de consolidação das boas práticas obstétricas e dos preceitos da Política de Humanização do Parto.

As mulheres revelaram que a imposição de condutas e ações mecanizadas compromete o direito à saúde e ao parto com qualidade, bem como a sua autonomia e poder decisório no cenário do cuidado. O profissional de enfermagem necessita estabelecer interações efetivas e relações de cuidado embasada na solidariedade, apoio emocional, conforto e calor humano, dada as circunstâncias do processo de parturição. Nessa perspectiva, a teoria de Watson possibilita refletir sobre a assistência de enfermagem obstétrica centrada no protagonismo do parto e na autonomia, beneficiando tomadas de decisões mais próximas dos desejos e anseios das mulheres e o fortalecimento de relações de cuidado empáticas.

Esperava-se outro cenário, capaz de influenciar positivamente na percepção de parto dessas mulheres, uma vez que esta pesquisa foi realizada em um hospital-escola. Assim, faz-se necessário um maior envolvimento e sensibilização dos gestores públicos, engajamento profissional e mais discussões no meio acadêmico e nos locais de atendimento a estas mulheres/gestantes/parturientes quanto ao exercício do empowerment no contexto obstétrico.

Por fim, mesmo este estudo sendo de uma realidade local, espera-se trazer contributos para o repensar de posturas e atitudes profissionais condizentes com as propostas da humanização, bem como a reorientação na formação do profissional de saúde, que ainda é delineada pela lógica do modelo biomédico. Partindo dessas proposições, é possível fortalecer as políticas públicas de assistência à saúde da mulher e ao parto, por meio de estratégias intersetoriais, e reunir elementos favoráveis a produção do cuidado em Enfermagem que contemple a mulher em sua integralidade.

\section{REFERÊNCIAS}

1. Velho MB, Oliveira ME, Santos EKA. Reflexões sobre a assistência de enfermagem prestada à parturiente. Rev Bras Enferm. 2010; 63(4): 652-659.

2. Almeida NAM, Medeiros M, Souza MR. Perspectivas de dor do parto normal de primigestas no período pré-natal. Texto contexto - enferm. 2012; 21(4): 819-827.

3. Velho MB, Santos EKA, Brüggemann OM, Camargo BV. Vivência do parto normal ou cesáreo: revisão integrativa sobre a percepção de mulheres. Texto contexto - enferm. 2012; 21(2): 458-466.

4. Gonçalves R, Aguiar CA, Merighi MAB, 
Jesus MCP. Vivenciando o cuidado no contexto de uma casa de parto: o olhar das usuárias. Rev Esc Enferm USP. 2011; 45(1): 62-70.

5. Watson J. Caring science as sacred science. Philadelphia: F.A. Davis; 2004. p. 129135.

6. Fontanella BJB, Ricas J, Turato, ER. Amostragem por saturação em pesquisas qualitativas em saúde: contribuições teóricas. Cad Saude Publica. 2008; 24(1): 17-27.

7. Bardin L. Análise de conteúdo. $3^{a}$ ed. Lisboa: Ed 70; 2008. p. 213-224.

8. Benute GRG, Nomura RY, Santos AM, Zarvos MA, Lucia MCS, Francisco RPV. Preferência pela via de parto: uma comparação entre gestantes nulíparas e primíparas. Rev Bras Ginecol Obstet. 2013; 35(6): 281-285.

9. Domingues RMSM, Dias MAB, Nakamura-Pereira M, Torres JA, D'Orsi E, Pereira APE et al. Processo de decisão pelo tipo de parto no Brasil: da preferência inicial das mulheres à via de parto final. Cad Saude Pública. 2014; 30(1): S101-S116.

10. Oliveira ASS, Rodrigues DP, Guedes MVC, Felipe GF. Percepção de mulheres sobre a vivência do trabalho de parto e parto. Rev. Rene. 2010; 11:32-41.

11. Leguizamon Junior T, Steffani JA, Bonamigo EL. Escolha da via de parto: expectativa de gestantes e obstetras. Rev. Bioét [Internet]. 2013 Dec [citado 15 jul 2015]; 21(3): 509-517. Disponível em: http://www.scielo.br/scielo.php?pi$\mathrm{d}=$ S1983-80422013000300015\&script $=$ - ci_abstract\&tlng $=\mathrm{pt}$

12. Pires D, Fertonani HP, Conill EM, Matos TA, Cordova FP, Mazur CS. A influência da assistência profissional em saúde na escolha do tipo de parto: um olhar sócio antropológico na saúde suplementar brasileira. Rev. Bras. Saude Mater. Infant [Internet]. 2010 [citado 15 jul 2015]; 10(2): 191-197. Disponível em: http://www. scielo.br/scielo.php?script=sci_arttex-
t\&pid=S1519-38292010000200006

13. Savieto RM, Leão ER. Nursing assistance and Jean Watson: a reflection on empathy. Esc Anna Nery. 2016; 20(1): 198-202.

14. D'Artibale EF, Bercini LO. Early contact and breastfeeding: meanings and experiences. Texto contexto - enferm, 2014; 23(1): 109-117.

15. Giraldo DI, González EM, Henao CP. Experiencias de las mujeres durante el trabajo de parto y parto. Av.enferm. [Internet]. 2015 [citado 13 abr 2015]; 33(2): 271-281. Disponível em: http://www. scielo.org.co/scielo.php?script=sci_abstract\&pid=S0121-45002015000200009

16. Rosa R, Martins FE, Gasperi BL, Monticelli M, Siebert ERC, Martins NM. Mãe e filho: os primeiros laços de aproximação. Esc Anna Nery. 2010; 14: 105-112.

17. Silva LS, Lucena AT, Amorim ES, Brandão Neto W, Nascimento ER. Asistencia al parto desde el punto de vista de las mujeres atendidas en un hospital público. Rev Cubana Enfermer [Internet]. 2014 Sep [citado 13 abr 2015]; 30(3): 192-203. Disponível em: http://new.medigraphic. com/cgi-bin/resumen.cgi?IDARTICU$\mathrm{LO}=62027$

18. D’Orsi E, Brüggemann OM, Diniz CSG, Aguiar JM, Gusman CR, Torres JA et al. Desigualdades sociais e satisfação das mulheres com o atendimento ao parto no Brasil: estudo nacional de base hospitalar. Cad Saude Pública. 2014; 30: S154-S168.

19. Ministério da Saúde $(\mathrm{Br})$. Portaria no 1020, de 29 de maio de 2013. Institui as diretrizes para a organização da Atenção à Saúde na Gestação de Alto Risco e define os critérios para a implantação e habilitação dos serviços de referência à Atenção à Saúde na Gestação de Alto Risco, incluída a Casa de Gestante, Bebê e Puérpera (CGBP), em conformidade com a Rede Cegonha [Internet]. Brasília: Ministério da Saúde; 2013 [citado 21 abr 2105]. Disponível em: http://bvsms. 
saude.gov.b/bvs/saudelegis/gm/2013/ prt1020_29_05_2013.html

20. Ministério da Saúde (Br). Secretaria de Políticas de Saúde. Parto, aborto e puerpério: assistência humanizada à mulher
[Internet]. Brasília: Ministério da Saúde; 2001 [citado 30 mar 2015]. 199 p. Disponível em: http://bvsms.saude.gov.br/bvs/ publicacoes/cd04_13.pdf 